\title{
Distribution of Trapped Magnetic Flux in Superconducting Stacks Magnetised by Angled Field
}

\author{
Lukasz Tomkow $^{1}$ (D) . Anis Smara ${ }^{1}$. Vicente Climente-Alarcon ${ }^{1} \cdot$ Bartek A. Glowacki $^{1}$ \\ Received: 30 October 2019 / Accepted: 27 November 2019/Published online: 18 December 2019 \\ (C) The Author(s) 2019
}

\begin{abstract}
Some novel energy applications require the use of complex shapes of stacks of superconducting tapes as trapped-flux magnets. A trapped-flux magnet magnetised in a superconducting motor may experience an angled magnetising field rather than a field normal to its surface. This will affect the trapped magnetic flux distribution. This work presents the results of the numerical and experimental analyses of the stacks magnetised in an angled magnetic field. The finite element model using H-formulation is developed to compute the induced superconducting currents. The measurements are performed on stacks with different thicknesses and with different orientations against a magnetising field. The resulting distribution of the magnetic flux as well as the electric currents is computed, presented and discussed in details. The importance of the observed distribution patterns is assessed in the context of the implementation of such stacks in a fully superconducting electric motor.
\end{abstract}

Keywords Superconducting tapes $\cdot$ Superconducting stacks $\cdot$ Magnetisation

\section{Introduction}

Stacked superconducting tapes can be applied as a very efficient trapped-flux magnet. Induction values as high as 17.7 $\mathrm{T}$ were achieved [1]. Thanks to low cost, simplicity and high thermal stability, they can outperform bulk superconductors in some applications with pulse magnetisation [2]. The stacks can be made of short pieces of tape, which otherwise would be unused, such as offcuts from production of Roebel cables [3, 4]. It is proposed to apply such magnets in a rotor of a superconducting synchronous motor for an aircraft [5]. The major advantage of the application of superconductors in an aircraft motor is the increase of power to weight ratio, achievable also by using the superconducting stator windings [6,7]. Other applications that may benefit from the decreased weight of the motors are marine propulsion [8] and wind turbines [9].

Lukasz Tomkow

1tt27@cam.ac.uk

1 Applied Superconductivity and Cryoscience Group, Department of Materials Science and Metallurgy, University of Cambridge, 27 Charles Babbage Road, Cambridge CB3 OFS, UK
The stacks applied as permanent magnets in a rotor, build as part of the motor for ASuMED project, are expected to have complex shapes, to optimise the performance of the motor and decrease demagnetisation [10-12]. Demagnetisation occurs mostly due to the external cross-fields, which change the patterns of currents present in the stack $[13,14]$. The issue of demagnetisation in a superconducting motor was analysed and it was found to significantly affect the operation of the machine and to decrease its power [15]. Power decay is further increased by heat generation [16].

This work addresses the issue of magnetisation of the superconducting stacks with magnetic fields, which are not perpendicular to the surface of the stack. Such fields will be present in the superconducting motor, both as magnetising and demagnetising fields. The fields will be distorted due to the inherent properties of the superconducting tapes. This distortion can lead to the changes in the performance of the motor and has to be considered when designing the stacks.

The obtained results are relevant also for different applications, such as levitation [17], or electric motors based on the diamagnetic behaviour of a bulk HTS [18]. The results can help understand the behaviour of tilted stacks, which were observed to provide good homogeneity and strength of the field [19]. Similar patterns of currents are observed in magnetic shields, which can be used to decrease 
demagnetisation [20], especially open magnetic shields made of short pieces of tapes [21].

The knowledge of the orientation of the magnetic flux in the stack can also help to assess and decrease the losses in the current leads made of stacks $[13,22]$. Such current leads can carry very large currents and benefit from improved thermal stability. Complex patterns of angled fields are expected to be formed in twisted stacks considered for the application in fusion reactors [23, 24].

In this paper, the strength and orientation of a trapped magnetic flux is analysed as the function of geometry of the stack and a magnetising filed. The effect of the thickness of the stack is investigated. The average angle of trapped magnetic field is calculated numerically. Strength of the trapped field is measured experimentally and calculated with a numerical model.

\section{Methods}

\subsection{Numerical}

Geometry of a numerical model is presented in Fig. 1. The model is divided into regions representing a stack, coils and environment modelled as air. The stack is always assumed to be horizontal in order to simplify the calculations and data analysis. In this model, the stack and the electromagnet have a common central point. The magnetising coils are rotated by a certain angle $\alpha$ with respect to the centre of the assembly.

A 2D numerical model is developed in Comsol Multiphysics. H-formulation is used to analyse the behaviour of the superconductor [25]. The stack is modelled as an anisotropic bulk to decrease the time of computations [26]. The main equation used is (1) [27].

$$
\frac{\partial H_{x}}{\partial t}+\frac{\partial H_{y}}{\partial t}+\frac{\partial}{\partial x}\left(E_{z}\left(J_{z}\right)\right)-\frac{\partial}{\partial y}\left(E_{z}\left(J_{z}\right)\right)=0
$$

$H$ is a local strength of magnetic field, $t$ is time, $E$ is electric field and $J$ is current density. $z$-component of electric field $E_{z}$ (out of model plane) is found with power law (2).

$E_{z}=\left\{\begin{array}{cr}E_{0}\left(\frac{\left|J_{z}\right|-J_{c}}{J_{c}}\right)^{n} \frac{J_{z}}{\left|J_{z}\right|} & \text { when }\left|J_{z}\right| \geq J_{c} \\ 0 & \text { when }\left|J_{z}\right|<J_{c}\end{array}\right.$

$J_{c}$ is critical current. $n$ is taken as 31 and $E_{0}$ is assumed as $100 \mu \mathrm{V} \cdot \mathrm{m}^{-1}[28,29] . J_{z}$ is found using (3).

$J_{z}=\frac{\partial H_{x}}{\partial y}-\frac{\partial H_{y}}{\partial x}$

Anisotropy of analysed tapes is considered based on [29]. Parameters $J_{c 0}$ and $B_{0}$ are fitted with data from [30] for SCS6050 tape. $J_{c}$ is found with (4).

$J_{c}=J_{c 0}\left[1+\epsilon{\frac{B}{B_{0}}}^{\alpha}\right]^{-\beta}$

$\alpha, \beta$ and $\gamma$ are $1,0.67$ and 2.77 respectively. $B$ is the local strength of magnetic induction and $\epsilon$ is found with (5). $J_{c 0}$ is normalised with the ratio between the width of the superconducting layer and the width of the entire tape. This leads to the decrease of critical current density by the factor of 100 .

$\epsilon=\sqrt{\gamma^{-2} \cdot\left(\frac{B_{x}}{B}\right)^{2}+\left(\frac{B_{y}}{B}\right)^{2}}$

The main value of interest in the case of a trapped-flux magnet is a total trapped flux $\Phi$. A component $k$ of $\Phi$ is calculated based on the results of simulations with (6). $L$ is the side of the stack and $\ell$ is a length element.

$\Phi_{k}=\oint_{L} B_{k} d \ell$

The angle of trapped flux $\alpha_{\Phi}$ is calculated using (7).

$\alpha_{\Phi}=\arccos \left(\frac{\Phi_{y}}{|\Phi|}\right)$

Numerical calculations are performed over the range of angles $\alpha$ between $0^{\circ}$ and $90^{\circ}$, with special focus on
Fig. 1 Geometry of the applied numerical model. Environment region of the model is not shown

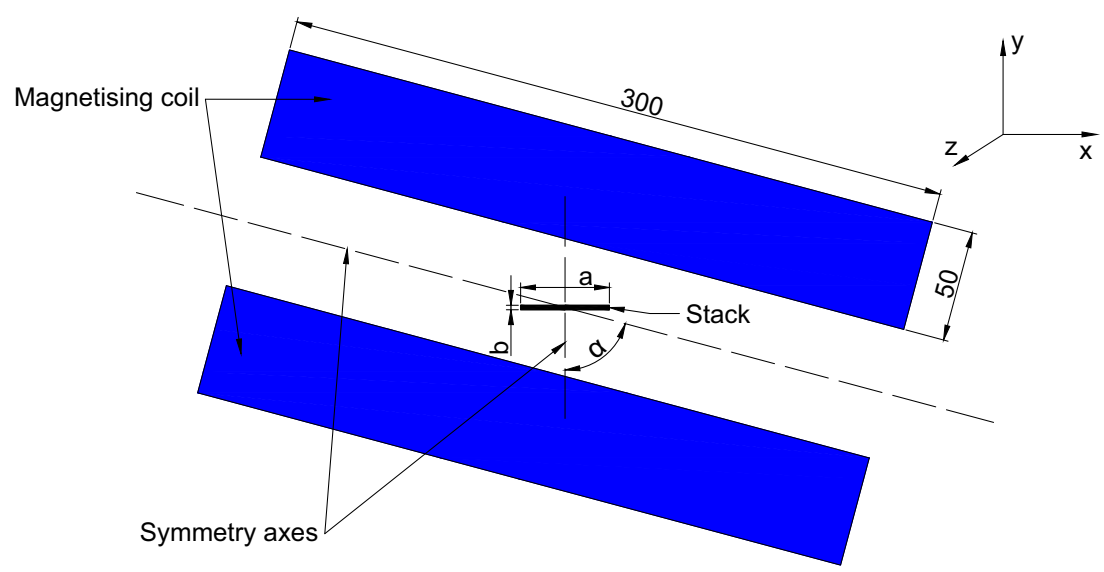


higher angles. The maximum magnetising induction with the considered geometry is approximately $3.4 \mathrm{~T}$, enough to fully magnetise the thinnest stack at $0^{\circ}$. This value is much higher than applied in experimental part and was selected to show the effect of full magnetisation on the properties of trapped field.

Additional numerical model is created to analyse experimental results. Geometry and strength of applied field reflect that used during the experiments. The numerical framework is the same, the only difference being the location of stack in relation to the coil. The axis of rotation of the coil is moved from the centre of the coil to its edge in the case of calculations with $45^{\circ}$ and $90^{\circ}$.

\subsection{Experimental}

Two stacks with 10 and 20 layers of superconducting tapes are made for experimental investigation. Each layer of the tape is approximately $100 \mu \mathrm{m}$ thick. Square pieces with the side length of $40 \mathrm{~mm}$ were cut from 40 -mm-wide superconducting tape from Deutsche Nanoschicht. The tape contains 1- $\mu \mathrm{m}$ layer of GdBCO on $70 \mu \mathrm{m}$ of NiW substrate and is coated on both sides with silver. The tapes in stacks are connected using Stycast 1266, impregnated in a vacuum chamber. The 20-layer stack is shown in Fig. 2c.

The measurements are performed using a scanning Hall probe magnetometry system, described at [19]. In the case of measurements with the angle of $0^{\circ}$, the sample is placed in a holder directly under the scanning probe, both visible at the bottom of Fig. 2a. Then, the entire assembly is placed inside the coil in a cooling vessel, shown in Fig. 2b. After achieving the desired temperature, the sample is magnetised and the measurements of the trapped field are performed. The probe is placed in position by the system of stepper motors visible in the middle of Fig. 2a. The magnetic induction is measured along the middle of the sample, over the span of $30 \mathrm{~mm}$, starting at the centre.

In the case of the measurements with other angles, the sample is secured on the side of the coil, as shown in Fig. 2b. Magnetisation is performed when only half of the stack is inside the coil. After magnetisation, the sample is moved to the horizontal position and the probing system is placed on the top to perform the measurements. Thanks to the construction of the holder, the measurements can be performed closer to the surface of the stack, than in the case with angle of $0^{\circ}$.

The samples are cooled in zero field conditions with liquid nitrogen to approximately $77 \mathrm{~K}$. Then, they are magnetised by a solenoidal coil fed with the current of $10 \mathrm{~A}$, corresponding with the applied field of approximately $113 \mathrm{mT}$. Results of measurements are then compared with the numerical calculations performed with parameters reflecting the conditions of the experiment. Measurements
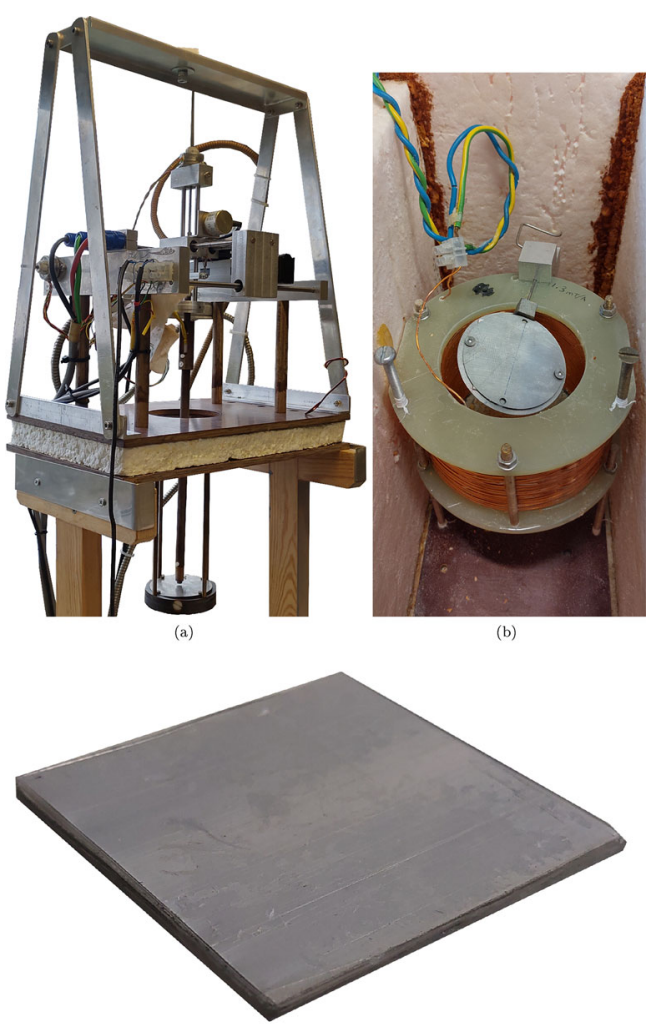

(c)

Fig. 2 Photographies of the measurement system. a Scanning probe assembly with a sample holder and a Hall probe visible at the bottom. b Coil in a cooling vessel with an angled field holder attached. c 20-layer stack

at $0^{\circ}$ for 10 and 20-layer stacks are used as reference cases to find parameter $J_{c 0}$, as the magnetisation is performed in the most homogeneous field.

\subsection{Results and Discussion}

Figure 3 shows two components of magnetic flux generated by a trapped-flux magnet after the removal of the magnetising flux. The values are normalised to the highest flux component for a given stack. The shape of the trapped flux is strongly influenced by the thickness of the stack. Thin stacks maintain the direction of the flux perpendicular to the surface in a wide range of angles of magnetising field. With the increase of the stack thickness, the dependence becomes closer to sinusoidal. The total trapped flux becomes weaker with the increase of the magnetising field angle. The relative decrease of the strength increases with the thickness of the stack.

Figure 4 shows the angle of the trapped flux against the angle of the magnetising field. With the increase of the stack thickness, the dependence slowly approaches the equal line. For the analysed geometries, the trapped field remains perpendicular to the surface for the majority of the 


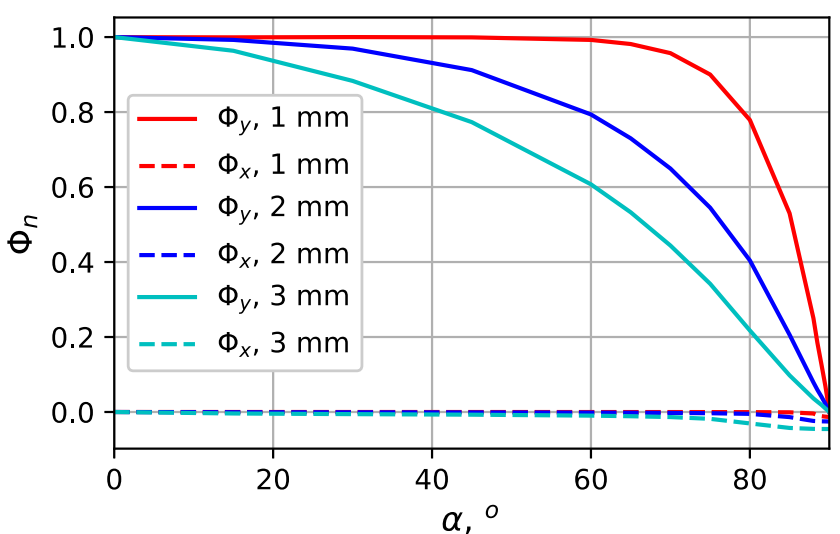

Fig. 3 Magnetic flux trapped by stacks with different thicknesses, normalised against the strongest flux for a given stack

considered range of angles of magnetising field. For the stack with the thickness/width ratio of $1 / 40$, the angle of trapped field starts to change significantly only if the angle of magnetising field exceeds $85^{\circ}$. For stack with the ratio of $3 / 40$, this value is approximately $65^{\circ}$.

Magnetisation per single tape is presented in Fig. 5. For each angle and strength of magnetising field, an optimal number of tapes exist, when the magnetisation per tape is maximum. This number decreases with the angle of applied field. The existence of such optimum is connected with the distribution of currents in a stack. The presented results are valid only for the analysed strength of the magnetising field and geometries of a stack. Experimental results presented further show that the optimum number of tapes increases with the increase of the magnetising field.

The effect of current distribution on the trapped flux is well visible in Fig. 6, showing the current density in the stack and strength of magnetic induction outside of it, along with magnetic field lines. At low angles, the thicker stack is not fully magnetised (for the given strength of

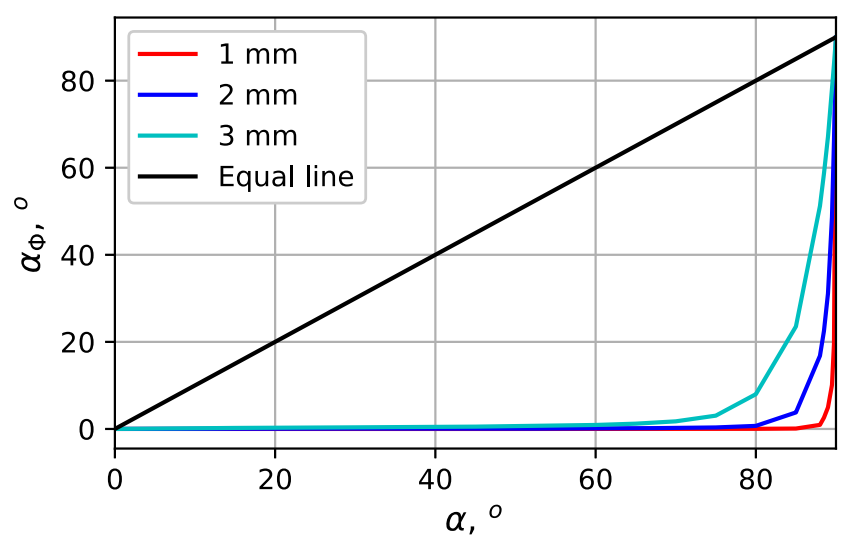

Fig. 4 Angle of magnetic flux $\alpha_{\Phi}$ (found with formula (7)), trapped by stacks with different thicknesses

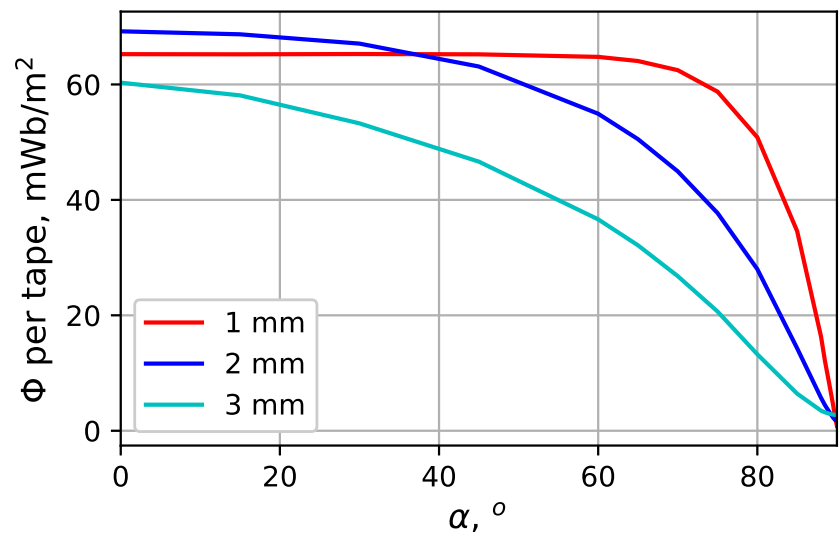

Fig. 5 Strength of trapped magnetic flux trapped in 40-mm-wide stack per single tape, per unit length, for stacks with different thicknesses. Each mm of stack thickness corresponds to 10 tapes

magnetic field), resulting in the formation of two current loops and the decrease of apparent magnetisation per single tape. In higher range of angles, the distortion of current is significantly stronger in larger stack, resulting in the shift of the field lines inside the stack.

Despite the shift inside of the stack, the magnetic induction lines outside of a stack remain mostly perpendicular to the surface at the lower angles of the magnetising field. When the angle of magnetising current reaches $90^{\circ}$, a single current loop is formed, generating magnetic flux parallel to the surface. The current in the loop is significantly weaker, than in the previously considered cases. This is due to the anisotropy of the stack. In the existing device, such situation probably would not happen, as the individual tapes have weak or no electrical connection and the current loops would be generated in individual tapes. However, in the case of a thin stack, their combined effect is expected to be similar to the homogenised.

The obtained results are important in the context of motor applications as they show that there exists an optimum mass/flux ratio of a stack. The number of tapes in a stack and its geometry can be selected to maximise the magnetisation per tape. In the optimal case, the magnetising field does not remain unused (what happens if a stack is too thin), and the magnetisation of the stack is close to full. If a stack is too thick, the magnetisation is only partial and some of its mass is left non-utilised.

Experimental measurements are performed in magnetic fields lower than modelled in previously described results. Therefore, the detrimental effect of partial magnetisation on the performance of a stack is even more pronounced. It is well visible in Fig. 7a, showing a component of trapped magnetic induction perpendicular to the stack, $5.5 \mathrm{~mm}$ above the centre of the sample. Total trapped magnetic flux decreases with the number of tapes, as the stack is only partially magnetised. Additionally, thermal effects affect 
Fig. 6 a-f Results of numerical calculations. Distribution of strength and direction of magnetic flux are shown outside of a stack region, coloured with yellow-red scale. Electric current density is shown inside the stack region, coloured with red-blue scale. The distributions are shown for different combinations of angles of magnetising field and thicknesses of the stacks

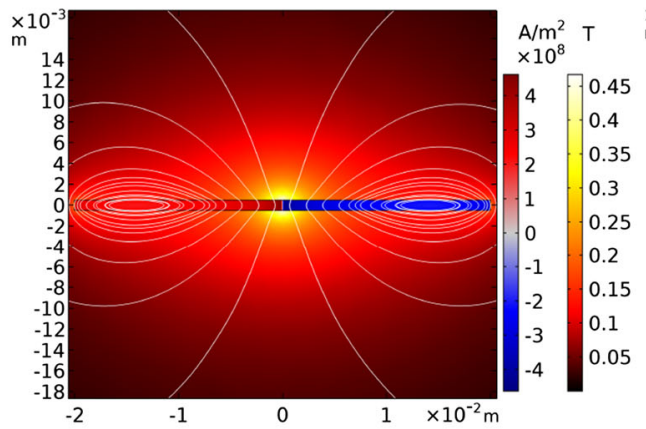

(a) $1 \mathrm{~mm}, 0^{\circ}$

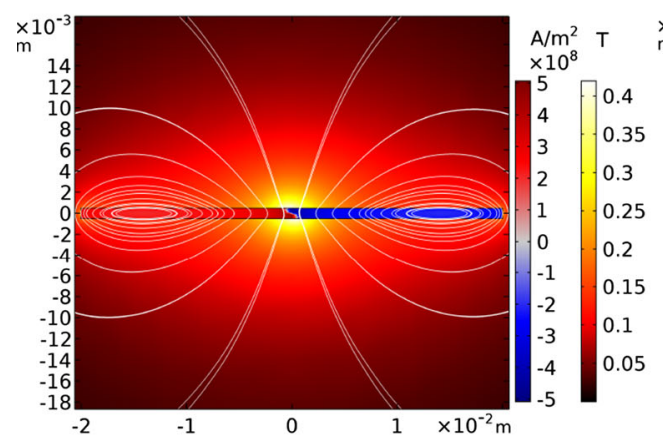

(c) $1 \mathrm{~mm}, 45^{\circ}$

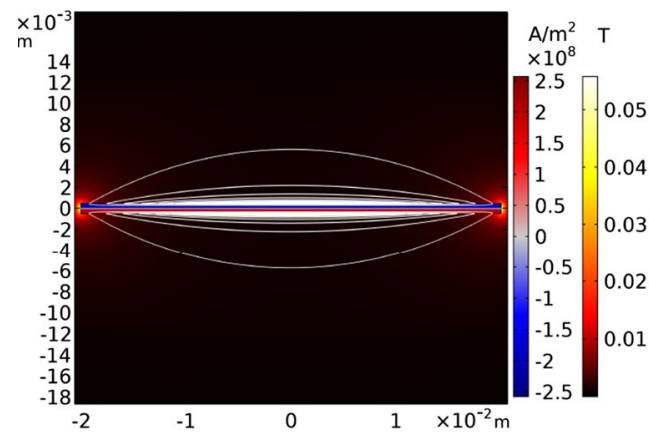

(e) $1 \mathrm{~mm}, 90^{\circ}$

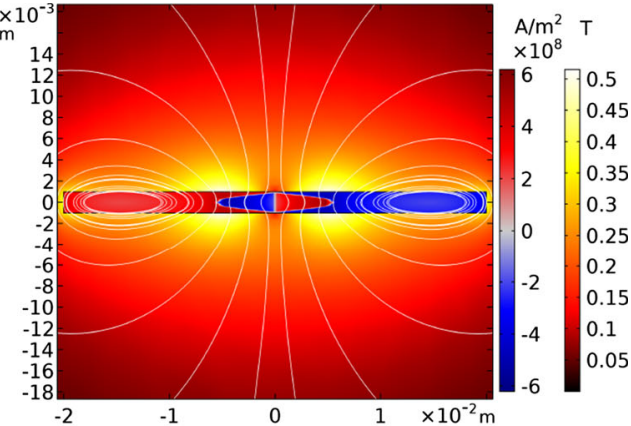

(b) $2 \mathrm{~mm}, 0^{\circ}$

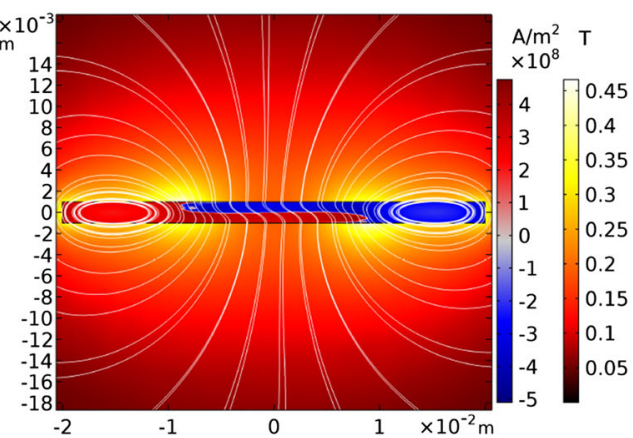

(d) $2 \mathrm{~mm}, 45^{\circ}$

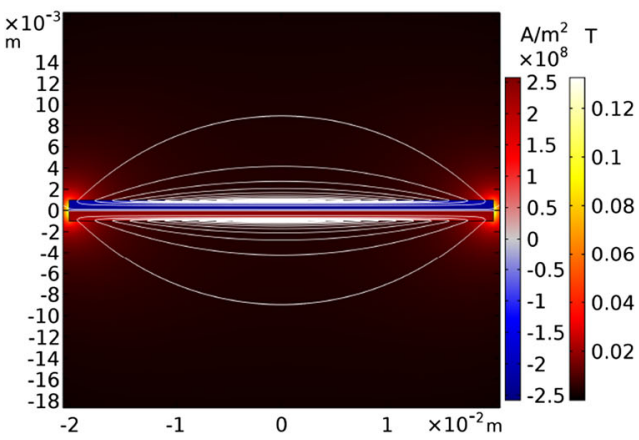

(f) $2 \mathrm{~mm}, 90^{\circ}$ critical current density, further exacerbating the situation. In the case of 10-layer stack, a very good agreement between numerical and experimental results is achieved with $J_{c 0}$ of $2.1 \cdot 10^{8} \mathrm{~A} \cdot \mathrm{m}^{-2}$. In the case of 20-layer stack, this value is $1.8 \cdot 10^{8} \mathrm{~A} \cdot \mathrm{m}^{-2}$, hinting at the decrease of $J_{c}$ caused by higher temperature.

Measurements in angled field were performed at the end of the coil; therefore, the stacks experienced a magnetising field with non-uniform strength. Additionally, due to the measurement method, the samples were placed slightly off the centre of the coil. Despite that, the measured and calculated trapped flux is surprisingly uniform, especially in the case of measurements performed in $90^{\circ}$, shown in Fig. 7c. The measured and calculated flux is slightly stronger than in the case of measurements in $45^{\circ}$ shown in Fig. $7 b$, seemingly contrary to previous modelling results. It has two reasons. Firstly, during the measurements in $45^{\circ}$, the magnetising field experienced by the stack is much weaker, because it does not reach as far into the coil, as in the other case. Secondly, the measuring probe was placed slightly closer in during the measurements in $90^{\circ}$.

While taking into consideration non-perfection of placement of the stack the magnetising field, the results of modelling and experiments agree well in all cases. The results show that even slight differences in the direction and strength distribution of the magnetising field can have significant effect on the trapped flux. It is especially important during manufacturing phase of a device using trapped-flux magnets. All possible distortions of the field should be considered, as well as the potential thermal effects, to find the most effective size and shape of the stack. 


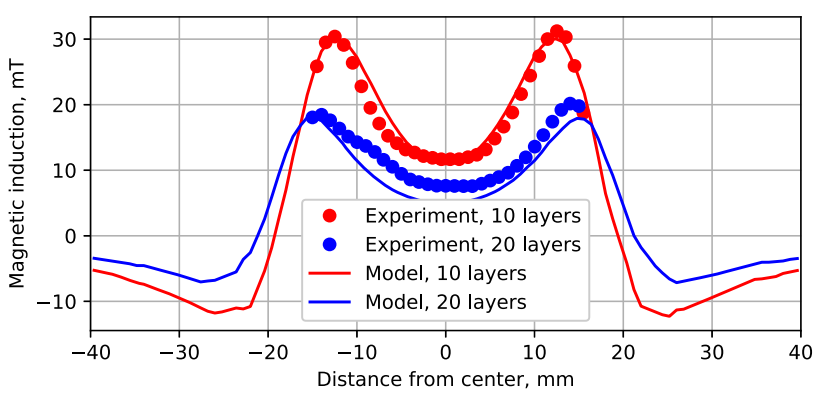

(a) $0^{\circ}$

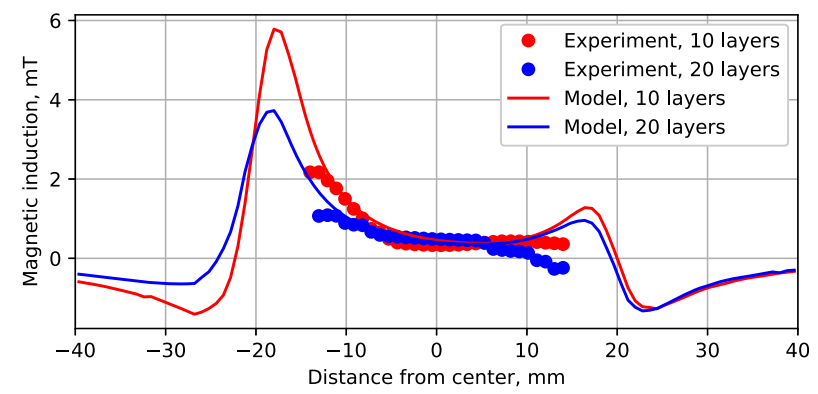

(b) $45^{\circ}$

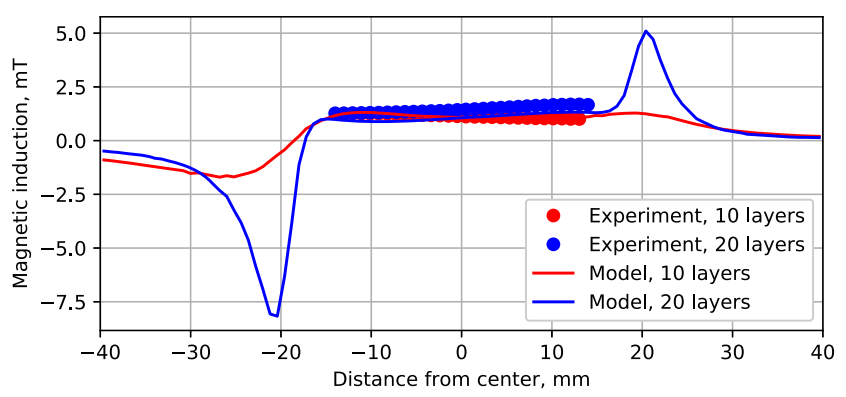

(c) $90^{\circ}$

Fig. 7 a-c Measured and modelled vertical component of the trapped field in $\mathrm{mT}$. The values are presented as the function of stack thickness and orientation

\section{Conclusions}

Numerical simulations and experimental measurements are performed to analyse the trapped magnetic flux in a stack of superconducting tapes. Different sizes of stacks and geometries of magnetising field are analysed. The results of the experiments and modelling are consistent. It is shown that at sufficiently low magnetising field, the trapped flux can decrease with the number of tapes, as parts of the stack are shielded by itself, and do not carry electric current.

If the magnetising field is strong enough, the total trapped magnetic flux increases with the number of tapes and decreases with the angle between a surface of a stack and a direction of the magnetising field. The strength of the trapped flux and perpendicularity are better maintained in thinner stacks. Magnetisation per single tape depends on angle and strength of the magnetising field, and the number of tapes in a stack. For each combination of strength and direction of the magnetising field, an optimum number of tapes exist, when the magnetisation per tape is the highest. Described considerations allow to find the optimum number of tapes in a stack for a given application.

During the design of a trapped-flux magnet, the issues of non-uniformity of the magnetising field have to be considered. The stacks display certain robustness in maintaining the direction of trapped flux, what is not always the desired outcome. Numerical simulations, including the relatively simplified method described in this paper, appear to be a reliable tool in the prediction of the behaviour of the stacks.

Funding Information This research is financially supported partially by the European Union's Horizon 2020 research innovation programme under grant agreement no. 7231119 (ASuMED "Advanced Superconducting Motor Experimental Demonstrator") and also by EPSRC grant no. EP/P000738/1 entitled "Development of superconducting composite permanent magnets for synchronous motors: an enabling technology for future electric aircraft".

\section{Compliance with Ethical Standards}

Conflict of Interest The authors declare that they have no conflict of interest.

Open Access This article is licensed under a Creative Commons Attribution 4.0 International License, which permits use, sharing, adaptation, distribution and reproduction in any medium or format, as long as you give appropriate credit to the original author(s) and the source, provide a link to the Creative Commons licence, and indicate if changes were made. The images or other third party material in this article are included in the article's Creative Commons licence, unless indicated otherwise in a credit line to the material. If material is not included in the article's Creative Commons licence and your intended use is not permitted by statutory regulation or exceeds the permitted use, you will need to obtain permission directly from the copyright holder. To view a copy of this licence, visit http://creativecommons. org/licenses/by/4.0/.

\section{References}

1. Patel, A., Baskys, A., Mitchell-Williams, T., McCaul, A., Coniglio, W., Hänisch, J., Lao, M., Glowacki, B.A.: Supercond. Sci. Technol. 31(9), 09LT01 (2018). https://doi.org/10.1088/13616668/aad34c

2. Baskys, A., Patel, A., Hopkins, S.C., Kalitka, V., Molodyk, A., Glowacki, B.A.: IEEE Trans. Appl. Supercond. 25(3), 1 (2015). https://doi.org/10.1109/TASC.2014.2360871

3. Goldacker, W., Grilli, F., Pardo, E., Kario, A., Schlachter, S., Vojenčiak, M.: Supercond. Sci. Technol. 27, 093001 (2014). https://doi.org/10.1088/0953-2048/27/9/093001

4. Mitchell-Williams, T.B., Patel, A., Baskys, A., Hopkins, S.C., Kario, A., Goldacker, W., Glowacki, B.A.: IEEE Trans. Appl. 
Supercond. 26(3), 1 (2016). https://doi.org/10.1109/TASC.2016. 2518994

5. Climente-Alarcon, V., Patel, A., Baskys, A., Glowacki, B.A.: IOP Conf. Series: Mater. Sci. Eng. 502, 012182 (2019). https://doi.org/10.1088/1757-899x/502/1/012182

6. Zanegin, S., Ivanov, N., Shishov, D., Shishov, I., Kovalev, K., Zubko, V.: Journal of Superconductivity and Novel Magnetism. https://doi.org/10.1007/s10948-019-05226-1 (2019)

7. Wen, C., Liu, J., Yu, Z., Liu, J., Zhao, Z., Wang, J.: Journal of Superconductivity and Novel Magnetism. https://doi.org/10.1007/ s10948-019-5113-5 (2019)

8. Kim, J.H., Park, S., Le, T.D., Jo, H.C., Jo, Y.S., Choi, Y.H., Lee, H., Kim, H.M.: J. Supercond. Nov. Magn. 28(2), 671 (2015). https://doi.org/10.1007/s10948-014-2810-y

9. Keysan, O., Olczak, D., Mueller, M.A.: J. Supercond. Nov. Magn. 26(5), 2103 (2013). https://doi.org/10.1007/s10948-012-1950-1

10. Patel, A., Climente-Alarcon, V., Baskys, A., Glowacki, B.A., Reis, T.: Design considerations for fully superconducting synchronous motors aimed at future electric aircraft. https://doi.org/10.1109/ESARS-ITEC.2018.8607734 (2018)

11. Baskys, A., Patel, A., Glowacki, B.A.: Supercond. Sci. Technol. 31(6), 065011 (2018). https://doi.org/10.1088/1361-6668/aabf32

12. Baskys, A., Patel, A., Climente-Alarcon, V., Glowacki, B.A.: Journal of Superconductivity and Novel Magnetism. https://doi.org/10.1007/s10948-019-5022-7 (2019)

13. Park, M., Choi, M., Hahn, S., Cha, G., Lee, J.: IEEE Trans. Appl. Supercond. 14(2), 1106 (2004). https://doi.org/10.1109/TASC. 2004.830429

14. Baghdadi, M., Ruiz, H.S., Coombs, T.A.: Sci. Rep. 8(1), 1342 (2018). https://doi.org/10.1038/s41598-018-19681-8

15. Smara, A., Mineev, N., Climente-Alarcon, V., Patel, A., Baskys, A., Glowacki, B.A., Reis, T.: Supercond. Sci. Technol. 32(8), 085009 (2019). https://doi.org/10.1088/1361-6668/ab20bf

16. Climente-Alarcon, V., Smara, A., Patel, A., Glowacki, B.A., Baskys, A., Reis, T.: In: AIAA Propulsion and Energy Forum and Exposition. Indianapolis, p. 3189332 (2019)

17. Anischenko, I.V., Pokrovskii, S.V., Mineev, N.A.: J. Phys.: Conf. Series 941, 012057 (2017). https://doi.org/10.1088/1742-6596/ 941/1/012057

18. Racz, A., Hadur, A., Vajda, I.: J. Supercond. Nov. Magn. 28(2), 663 (2015). https://doi.org/10.1007/s 10948-014-2792-9
19. Mitchell-Williams, T.B., Baskys, A., Hopkins, S.C., Kalitka, V., Molodyk, A., Glowacki, B.A., Patel, A.: Supercond. Sci. Technol. 29(8), 085008 (2016). https://doi.org/10.1088/0953-2048/29/8/ 085008

20. Baghdadi, M., Ruiz, H.S., Fagnard, J.F., Zhang, M., Wang, W., Coombs, T.A.: IEEE Trans. Appl. Supercond. 25(3), 1 (2015). https://doi.org/10.1109/TASC.2014.2372873

21. Tomkow, L., Kulikov, E., Kozlowski, K., Drobin, V.: J. Appl. Phys. 126(8), 083903 (2019). https://doi.org/10.1063/1.5112036

22. Diev, D., Galimov, A., Ilin, A., Khodzhibagiyan, H., Kovalev, I., Makarenko, M., Naumov, A., Novikov, M., Novikov, S., Polyakov, A., Shcherbakov, V., Shevchenko, S., Shutova, D., Surin, M.: Cryogenics 94, 45 (2018). https://doi.org/10.1016/j. cryogenics.2018.07.006. http://www.sciencedirect.com/science/ article/pii/S0011227518301553

23. Bykovsky, N., Uglietti, D., Wesche, R., Bruzzone, P.: IEEE Trans. Appl. Supercond. 26(2), 1 (2016). https://doi.org/10.1109/TASC. 2016.2517187

24. Bykovsky, N., Marzi, G.D., Uglietti, D., Bruzzone, P., Muzzi, L.: Supercond. Sci. Technol. 30(2), 024010 (2016). https://doi.org/10. 1088/1361-6668/30/2/024010

25. Pecher, R., McCulloch, M., Chapman, S., Prigozhin, L.: Proc EUCAS (2003)

26. Zermeno, V.M.R., Abrahamsen, A.B., Mijatovic, N., Jensen, B.B., Sørensen, M.P.: J. Appl. Phys. 114(17), 173901 (2013). https://doi.org/10.1063/1.4827375

27. Zhang, M., Kvitkovic, J., Kim, J.H., Kim, C.H., Pamidi, S.V., Coombs, T.A.: Appl. Phys. Lett. 101(10), 102602 (2012). https://doi.org/10.1063/1.4749275

28. Kvitkovic, J., Patel, S., Zhang, M., Zhang, Z., Peetz, J., Marney, A., Pamidi, S.: IEEE Trans. Appl. Supercond. 28(4), 1 (2018). https://doi.org/10.1109/TASC.2018.2813538

29. Zhang, X., Zhong, Z., Geng, J., Shen, B., Ma, J., Li, C., Zhang, H., Dong, Q., Coombs, T.A.: Journal of Superconductivity and Novel Magnetism. https://doi.org/10.1007/s10948-018-4678-8 (2018)

30. Zhang, M., Kim, J.H., Pamidi, S., Chudy, M., Yuan, W., Coombs, T.A.: J. Appl. Phys. 111(8), 083902 (2012). https://doi.org/10. $1063 / 1.3698317$

Publisher's Note Springer Nature remains neutral with regard to jurisdictional claims in published maps and institutional affiliations. 